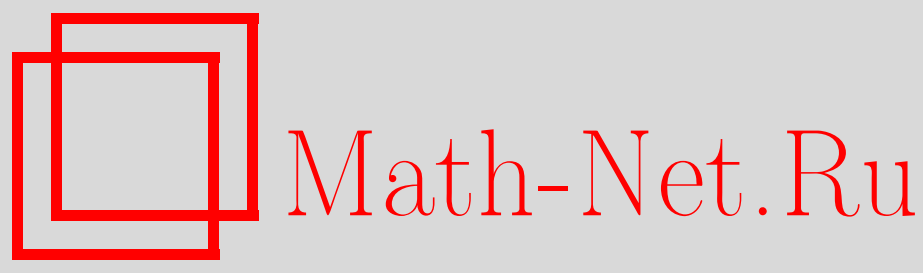

Г. М. Жислин, Принцип Паули, устойчивость и связанные состояния систем тождественных псевдорелятивистских частиц, ТМФ, 2008, том 157, номер 1, 116-129

DOI: https://doi.org/10.4213/tmf6267

Использование Общероссийского математического портала Math-Net.Ru подразумевает, что вы прочитали и согласны с пользовательским соглашением http://www.mathnet.ru/rus/agreement

Параметры загрузки:

IP : 34.239 .49 .27

26 апреля 2023 г., $16: 44: 48$

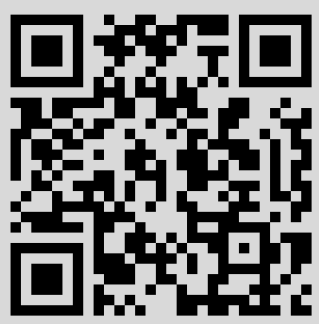




\title{
ПРИНЦИП ПАУЛИ, УСТОЙЧИВОСТЬ И СВЯЗАННЫЕ СОСТОЯНИЯ СИСТЕМ ТОЖДЕСТВЕННЫХ ПСЕВДОРЕЛЯТИВИСТСКИХ ЧАСТИЦ
}

\begin{abstract}
На основе анализа свойств псевдорелятивистских гамильтонианов систем $Z_{n}$ из $n$ тождественных частиц установлено, что для реальных (короткодействующих) потенциалов взаимодействия между частицами существует бесконечная последовательность таких чисел $n_{s}, s=1,2, \ldots$, что система $Z_{n_{s}}$ устойчива, причем $\sup _{s} n_{s+1} n_{s}^{-1}<+\infty$. Для устойчивых систем $Z_{n}$ доказано, что гамильтониан относительного движения таких систем имеет непустой дискретный спектр при некоторых фиксированных значениях полного момента частиц. Результаты получены с полным учетом перестановочной симметрии (запрета Паули) как для фермионных, так и для бозонных систем при любом значении спина частиц. Ранее для псевдорелятивистских систем подобные утверждения были доказаны только без учета перестановочной симметрии и поэтому не имели физического смысла; для нерелятивистских систем результаты с учетом перестановочной симметрии (но без оценки отношения $n_{s+1} n_{s}^{-1}$ ) имелись, но были получены в предположениях, справедливость которых для реальных систем до сих пор не установлена. Основная теорема верна и для нерелятивистских систем, что существенно усиливает имевшийся результат.
\end{abstract}

Ключевые слова: псевдорелятивистские системы, устойчивость, принцип Паули, дискретный спектр, многочастичные гамильтонианы.

\section{1. ВВЕДЕНИЕ}

1.1. В настоящей работе на основе изучения свойств псевдорелятивистских (ПР) гамильтонианов исследуются возможность существования устойчивых ПР-систем $Z_{n}$, состоящие из $n$ тождественных частиц, при $n \rightarrow \infty$ и вопрос о существовании непустого дискретного спектра операторов энергии относительного движения устойчивых ПР-систем. Установлено, что для реальных (короткодействующих) потенциалов взаимодействия между частицами существует бесконечная последовательность таких чисел $n_{s}, s=1,2, \ldots$, что система $Z_{n_{s}}$ устойчива, при этом $\sup _{s} n_{s+1} n_{s}^{-1}<+\infty$ (теорема 2.2). Для устойчивых систем $Z_{n}$ доказано, что гамильтониан относительного движения такой системы имеет непустой дискретный спектр при некоторых

\footnotetext{
* Федеральное государственное научное учреждение "Научно-исследовательский радиофизический институт", Н. Новгород, Россия. E-mail: greg@nirfi.sci-nnov.ru
} 
фиксированных значениях полного момента частиц системы $Z_{n}$ (теорема 2.1). Все результаты получены с полным учетом перестановочной симметрии (запрета Паули) как для фермионных, так и для бозонных систем при любом значении спина частиц.

Ранее для ПР-систем подобные утверждения были доказаны только без учета перестановочной симметрии [1] и поэтому не имели физического смысла. Для нерелятивистских (НP) систем результаты с учетом перестановочной симметрии (но без оценки отношения $n_{s+1} n_{s}^{-1}$ ) можно найти в работе [2], но там они были получены в таких предположениях, справедливость которых для реальных систем до сих пор не установлена (и которые в настоящей работе отсутствуют). В связи с этим отметим, что наша основная теорема 2.2 в той же формулировке верна для НР-систем, т.е. существенно усиливает результат работы [2].

1.2. Рассмотрим квантовую систему $Z_{n}$, состоящую из $n$ тождественных частиц. Пусть $r_{i}=\left(x_{i}, y_{i}, z_{i}\right)$ и $m$ - радиус-вектор и масса $i$-й частицы, $r=\left(r_{1}, \ldots, r_{n}\right)$. Тогда псевдорелятивистский оператор энергии системы $Z_{n}$ можно записать в виде

$$
H_{n}=K_{n}(r)+V_{n}(r),
$$

где $\mathrm{e}^{1)}$

$$
\begin{aligned}
K_{n}(r) & =\sum_{i=1}^{n} K\left(r_{i}\right), & V_{n}(r) & =\frac{1}{2} \sum_{i, j=1}^{n} V\left(\left|r_{i}-r_{j}\right|\right), \\
K\left(r_{i}\right) & =\sqrt{-\Delta_{i}+m^{2}}, & \Delta_{i} & =\frac{\partial^{2}}{\partial x_{i}^{2}}+\frac{\partial^{2}}{\partial y_{i}^{2}}+\frac{\partial^{2}}{\partial z_{i}^{2}},
\end{aligned}
$$

$V\left(\left|r_{i}\right|\right)$ - вещественная функция, $V\left(\left|r_{i}\right|\right) \in \mathcal{L}_{2, \text { lос }}\left(\mathbb{R}^{3}\right), V\left(\left|r_{i}\right|\right) \rightarrow 0$ при $\left|r_{i}\right| \rightarrow \infty$, причем функция $V\left(\left|r_{i}\right|\right)$ такова, что оператор $H_{n}$ ограничен снизу при любом $n$.

Одночастичные НР-операторы $K\left(r_{i}\right)$ суть интегральные операторы в координатном пространстве, однако в импульсном представлении они превращаются в операторы умножения. Действительно, пусть $\mathcal{F}$ - фурье-преобразование в $\mathbb{R}^{3}$ и $\bar{f}\left(p_{i}\right)=\mathcal{F}\left\{f\left(r_{i}\right)\right\}$, где $f\left(r_{i}\right) \in \mathcal{L}_{2}\left(\mathbb{R}^{3}\right)$ и $p_{i}$ - фурье-сопряженная координата к $r_{i}$. Тогда

$$
K\left(r_{i}\right) f\left(r_{i}\right)=\mathcal{F}^{-1}\left\{\sqrt{p_{i}^{2}+m^{2}} \bar{f}\left(p_{i}\right)\right\} .
$$

Положим $p=\left(p_{1}, \ldots, p_{n}\right)$,

$$
\bar{K}\left(p_{i}\right)=\sqrt{p_{i}^{2}+m^{2}}, \quad \bar{K}_{n}(p)=\sum_{i=1}^{n} \bar{K}\left(p_{i}\right)
$$

и используем далее наряду с (1.1) смешанную форму записи для оператора $H_{n}$ :

$$
H_{n}=\bar{K}_{n}(p)+V_{n}(r),
$$

где релятивистский оператор кинетической энергии $\bar{K}_{n}(p)$ рассматривается в импульсном представлении, а НР-оператор потенциальной энергии $V_{n}$ - в координатном.

1) Система единиц выбрана так, что константа Планка и скорость света равны 1. 
1.3. Чтобы определить понятие устойчивости системы $Z_{n}$, необходимо рассмотреть всевозможные ее разбиения и их операторы. Обозначим через $D=\left\{C_{1}, \ldots, C_{s}\right\}$ произвольное разбиение системы $Z_{n}$ на $s$ непересекающихся и не взаимодействующих друг с другом кластеров $C_{j}$ :

$$
C_{i} \cap C_{j}=\varnothing \quad \text { при } \quad i \neq j, \quad \bigcup_{j=1}^{s} C_{j}=Z_{n}, \quad 2 \leqslant s \leqslant n .
$$

Оператор энергии составной системы $D$ имеет вид

$$
H_{n}(D)=\sum_{j=1}^{s} H\left[C_{j}\right]
$$

где $H\left[C_{j}\right]-$ ПР-оператор энергии кластера $C_{j}$,

$$
H\left[C_{j}\right]=\sum_{i \in C_{j}} \bar{K}\left(p_{i}\right)+\frac{1}{2} \sum_{\substack{i, j \in C_{j}, i \neq j}} V\left(\left|r_{i}-r_{j}\right|\right) .
$$

Операторы $H_{n}$ и $H_{n}(D)$ ограничены снизу на $C_{0}\left(\mathbb{R}^{3 n}\right)$. Расширим их до самосопряженных, сохраняя прежние обозначения.

1.4. Операторы $H_{n}$ и $H_{n}(D)$ отвечают энергии полного движения соответственно системы $Z_{n}$ и составной системы $D$, и они вполне пригодны (после учета принципа Паули) для изучения стабильности системы $Z_{n}$. Однако, исследуя вопрос о структуре спектра связанных состояний $Z_{n}$, надо рассматривать оператор энергии относительного движения. При построении такого оператора в ПР-случае мы следуем работе [3]. Введем радиус-вектор $r_{0}=\sum_{j=1}^{n} r_{j} / n$ центра масс системы $Z_{n}$ и относительные координаты $q_{i}=r_{i}-r_{0}$ частиц. Фурье-сопряженные к ним координаты будут соответственно

$$
Q=\sum_{i=1}^{n} p_{i}
$$

и $p_{i}$, где $p_{i}$ - те же, что для $r_{i}, i=1,2, \ldots, n$. В новых координатах потенциал $V_{n}$ будет зависеть только от $q_{1}, \ldots, q_{n}$, а оператор кинетической энергии $\bar{K}_{n}$ - от $p_{1}, \ldots, p_{n}$ при условии (1.9). Если теперь фиксировать $Q$, то полученный оператор $H_{n}(Q)$ будет зависеть только от относительных координат $q_{i}$ и соответствующих импульсов $p_{i}$ (и от фиксированного $Q$ ). Поэтому $H_{n}(Q)$ можно рассматривать как оператор энергии относительного движения системы $Z_{n}$. Оператор $H_{n}(D)$ после введения координат $q_{i}, p_{i}, i=1,2, \ldots, n$, и фиксации $Q$ обозначим через $H_{n}(Q ; D)^{2)}$. Положим $R_{0}=\left\{q \mid q=\left(q_{1}, \ldots, q_{n}\right), q_{1}+q_{2}+\cdots+q_{n}=0\right\}$. Операторы $H_{n}(Q)$ и $H_{n}(Q ; D)$ будут рассматриваться в $\mathcal{L}_{2}\left(R_{0}\right)$.

\footnotetext{
2) Оператор $H_{n}(Q ; D)$ в отличие от $H_{n}(Q)$ не является оператором энергии относительного движения системы $D$, ибо не фиксированы значения моментов кластеров $C_{j}$, входящих в $D$, однако для нас это несущественно.
} 
1.5. Пусть $S_{n}$ - группа перестановок $n$ элементов и $E_{n}=\{\alpha\}$ - множество всех типов $\alpha$ неприводимых представлений группы $S_{n}$. Как известно [4], каждое $\alpha$ из $E_{n}$ однозначно определяется каким-либо разбиением числа $n$ на целые положительные числа:

$$
n=n_{1}+n_{2}+\cdots+n_{p}, \quad 1 \leqslant p \leqslant n,
$$

т.е. $\alpha=\alpha\left(n_{1}, \ldots, n_{p}\right)$. Пусть $k$ - произвольное целое число, $1 \leqslant k \leqslant n$. Положим

$$
\begin{aligned}
& E_{n}^{(1)}(k)=\left\{\alpha \mid \alpha \in E_{n}, \alpha=\alpha\left(n_{1}, \ldots, n_{p}\right), n_{i} \leqslant k, i=1,2, \ldots, p\right\}, \\
& E_{n}^{(2)}(k)=\left\{\alpha \mid \alpha \in E_{n}, \alpha=\alpha\left(n_{1}, \ldots, n_{p}\right), p \leqslant k\right\} .
\end{aligned}
$$

Используя для описания типов $\alpha \in E_{n}^{(i)}(k)$ схемы Юнга [5], можно сказать, что множества $E_{n}^{(1)}(k)$ и $E_{n}^{(2)}(k)$ состоят из тех $\alpha$, которые отвечают схемам Юнга, имеющим не более $k$ столбцов (для $\left.E_{n}^{(1)}(k)\right)$ или не более $k$ строк (для $E_{n}^{(2)}(k)$ ). Далее всюду полагаем $E_{n}(k)=E_{n}^{(1)}(k)$ или $E_{n}(k)=E_{n}^{(2)}(k)$. Обозначим через $P_{n}^{(\alpha)}$ проектор в $\mathcal{L}_{2}\left(\mathbb{R}^{3 n}\right)$ и $\mathcal{L}_{2}\left(R_{0}\right)$ соответственно на подпространства таких функций $\{\psi(r)\}$ и $\{\varphi(q)\}$, которые под действием операторов $T_{g}$,

$$
T_{g} \psi(r)=\psi\left(g^{-1} r\right), \quad T_{g} \varphi(q)=\varphi\left(g^{-1} q\right), \quad g \in S_{n},
$$

преобразуются по представлениям типа $\alpha$. Явный вид оператора $P_{n}^{(\alpha)}$ дан в п. 3.6. Положим

$$
B_{n}(k)=\sum_{\alpha \in E_{n}(k)} \bigoplus P_{n}^{(\alpha)} \mathcal{L}_{2}\left(\mathbb{R}^{3 n}\right), \quad B_{n, 0}(k)=\sum_{\alpha \in E_{n}(k)} P_{n}^{(\alpha)} \mathcal{L}_{2}\left(R_{0}\right)
$$

и обозначим через $H_{n}(k), H_{n}(D ; k)$ и $H_{n}(Q ; k), H_{n}(Q ; D ; k)$ ограничения операторов $H_{n}, H_{n}(D)$ и $H_{n}(Q), H_{n}(Q ; D)$ соответственно на пространства $B_{n}(k)$ и $B_{n, 0}(k)$. Пусть далее $A^{(\alpha)}=P_{n}^{(\alpha)} A$ для $A=H_{n}, H_{n}(Q), H_{n}(D), H_{n}(Q ; D)$. Очевидно, что

$$
\begin{aligned}
H_{n}(k) & =\sum_{\alpha \in E_{n}(k)} H_{n}^{(\alpha)}, & H_{n}(D ; k) & =\sum_{\alpha \in E_{n}(k)} H_{n}^{(\alpha)}(D), \\
H_{n}(Q ; k) & =\sum_{\alpha \in E_{n}(k)} H_{n}^{(\alpha)}(Q), & H_{n}(Q ; D ; k) & =\sum_{\alpha \in E_{n}(k)} H_{n}^{(\alpha)}(Q ; D) .
\end{aligned}
$$

1.6. Чтобы результаты данного исследования имели физический смысл, надо учесть запрет Паули. А это означает, следующее [6]:

во-первых, мы должны взять $E_{n}(k)=E_{n}^{(1)}(k)$, если система $Z_{n}$ состоит из фермионов, и $E_{n}(k)=E_{n}^{(2)}(k)$, если $Z_{n}$ состоит из бозонов;

во-вторых, число $k$ должно соответствовать возможным значениям спина частиц. Например, если рассматривать фермионы со спином $\pm 1 / 2$, то физический смысл имеют операторы $H_{n}(k), H_{n}(Q ; k)$ только при $E_{n}(k)=E_{n}^{(1)}(k), k=1,2$.

Однако, поскольку математическая формулировка результатов и их доказательство не зависят от указанных условий, далее мы не предполагаем обязательного их выполнения. В то же время всегда, когда мы будем использовать физические пояснения математических утверждений и определений, эти пояснения автоматически будут относиться только к ситуации, в которой данные требования выполнены. 


\section{2. УСТОЙЧИВОСТЬ И ДИСКРЕТНЫЙ СПЕКТР: ОСНОВНЫЕ РЕЗУЛЬТАТЫ}

2.1. Положим

$$
\mu_{n}(k)=\min _{D} \inf H_{n}(D ; k)
$$

Будем говорить, что система $Z_{n}$

$$
\begin{aligned}
k \text {-устойчива, если } & \inf H_{n}(k)<\mu_{n}(k), \\
k \text {-неустойчива, если } & \inf H_{n}(k) \geqslant \mu_{n}(k)
\end{aligned}
$$

(можно доказать, что строгое неравенство в (2.3) невозможно, но для нас это не существенно).

Так как оператор $H_{n}$ представляет собой ПР-оператор энергии системы $Z_{n}$, неравенство в (2.2) означает, что любое разбиение системы $Z_{n}$ приводит к возрастанию минимального значения энергии в пространстве состояний $B_{n}(k)$. Таким образом, $k$-устойчивость системы $Z_{n}$ эквивалентна энергетической невыгодности ее разбиений по отношению к состояниям из $B_{n}(k)$, т.е. эквивалентна устойчивости в обычном смысле, но в пространстве $B_{n}(k)$. При этом, если взять за $k$ максимальное из разрешенных принципом Паули значений, то можно говорить об устойчивости системы $Z_{n}$, не упоминая $k$. Однако использование понятия $k$-устойчивости, вообще говоря, позволяет получить дополнительную информацию о типе перестановочной симметрии устойчивых состояний. Чтобы понять причины этого, достаточно заметить, что $k$-устойчивость системы $Z_{n}$ не влечет за собой автоматически ее $k_{1}$-устойчивость при $k_{1} \neq k$. Действительно, пусть система $Z_{n} k$-устойчива и $k_{1} \neq k$. Для определенности считаем $k_{1}>k$. Тогда, поскольку $E_{n}(k) \subset E_{n}\left(k_{1}\right)$, имеем

$$
B_{n}(k) \subset B_{n}\left(k_{1}\right) .
$$

Поэтому

$$
\inf H_{n}\left(k_{1}\right) \leqslant \inf H_{n}(k), \quad \mu_{n}\left(k_{1}\right) \leqslant \mu_{n}(k) .
$$

Так как система $Z_{n} \quad k$-устойчива,

$$
\inf H_{n}(k)<\mu_{n}(k),
$$

и мы видим, что соотношений (2.5), (2.6) недостаточно для того, чтобы было справедливо неравенство

$$
\inf H_{n}\left(k_{1}\right)<\mu_{n}\left(k_{1}\right),
$$

т.е. для $k_{1}$-устойчивости системы $Z_{n}$. Поэтому мы не можем исключить ситуацию, когда система $Z_{n}$ одновременно $k$-устойчива и $k_{1}$-неустойчива при $k_{1} \neq k$.

2.2. Введем множество

$$
F_{n}(k)=\left\{\alpha \mid \alpha \in E_{n}(k), \inf H_{n}^{(\alpha)}<\mu_{n}(k)\right\} .
$$

Если система $Z_{n} k$-устойчива, то в силу $(1.12 \mathrm{a})$ и $(2.2)$ множество $F_{n}(k)$ не пусто. 
Теорема 2.1. Пусть система $Z_{n} \quad k$-устойчива. Тогда для любого $\alpha \in F_{n}(k)$ дискретный спектр $s_{\mathrm{d}}\left(H_{n}^{(\alpha)}(Q)\right)$ оператора $H_{n}^{(\alpha)}(Q)$ не пуст при всех $Q$ из некоторого множества $\Gamma_{n}(\alpha) \subseteq \mathbb{R}^{3}$.

СлеДСТВИЕ. Дискретный спектр $s_{\mathrm{d}}\left(H_{n}(Q ; k)\right)$ оператора $H_{n}(Q ; k) k$-yстойчивой системы $Z_{n}$ не пуст при $Q \in \Gamma_{n}[k]=\bigcup_{\alpha \in F_{n}(k)} \Gamma_{n}(\alpha)$.

ЗАмЕчАНИЕ 2.1. В НР-случае согласно ХВЖК-теореме устойчивость системы эквивалентна существованию непустого дискретного спектра оператора $\mathcal{H}$ энергии относительного движения при любом $Q$, ибо в НР-ситуации движение центра масс полностью отделяется и $\mathcal{H}$ не зависит от $Q$. В ПР-случае такое отделение невозможно, и поэтому структура дискретного спектра может зависеть от $Q^{3)}$.

2.3. Приведенная ниже теорема 2.2, составляющая основной результат настоящей статьи, говорит о том, что для широкого класса потенциалов взаимодействия (включающего наиболее интересные для приложений случаи) не существует ограничения сверху на число $n$ частиц в $k$-устойчивых системах $Z_{n}$.

Tеорема 2.2. Пусть существует такое $A>0$, что для куба $C(A) \subset \mathbb{R}^{3} c$ центром в начале координат и ребрами длины $2 A$, параллельными осям координат, выполняется неравенство

$$
\delta: \equiv \int_{r_{1}, r_{2} \in C(A)} V\left(\left|r_{1}-r_{2}\right|\right) d r_{1} d r_{2}<0 .
$$

Тогда для каждого $k \geqslant 1$ существует бесконечная последовательность таких чисел $n_{1}<n_{1}<\cdots<n_{s}<\cdots$, что системы $Z_{n_{s}}, s=1,2, \ldots, k$-устойчивы. Если дополнительно предположить, что

$$
d_{0}:=\inf _{r_{1}} V\left(\left|r_{1}\right|\right)>-\infty
$$

mo

$$
\sup _{s} \frac{n_{s+1}}{n_{s}}<+\infty
$$

ЗАмЕчАНИЕ 2.2. Для того чтобы имело место неравенство (2.8), достаточно выполнения условий [2]

$$
V\left(\left|r_{1}\right|\right) \in \mathcal{L}_{1}\left(\mathbb{R}^{3}\right), \quad \int_{\mathbb{R}^{3}} V\left(\left|r_{1}\right|\right) d r_{1}<0 .
$$

Эти условия выполняются, например, когда $V\left(\left|r_{1}\right|\right)$ есть линейная комбинация потенциалов Юкавы:

$$
V\left(\left|r_{1}\right|\right)=\sum_{i=1}^{m} a_{i} \frac{e^{-b_{i}\left|r_{1}\right|}}{\left|r_{1}\right|}, \quad b_{i}>0
$$

если

$$
\sum_{i=1}^{m} a_{i}>0, \quad \sum_{i=1}^{m} a_{i} b_{i}^{2}<0 .
$$

\footnotetext{
${ }^{3)}$ Например, для двухчастичной ПР-системы дискретный спектр оператора $H_{2}(Q)$ при некоторых потенциалах взаимодействия конечен для одних значений $Q$ и бесконечен для других [7].
} 
ЗАмЕчАниЕ 2.3. Утверждения теоремы 2.2 и ее доказательство практически без изменений переносятся на случай НР-систем.

2.4. Доказательства теорем 2.1, 2.2 даны в разделе 3 , а здесь мы сформулируем вопросы, на которые нам ответить не удалось.

1. Зависят ли "магические" числа $n_{1}, n_{2}, \ldots$ от $k$ ?

2 . Вытекает ли из $k$-устойчивости системы $Z_{n}$ ее $k_{1}$-устойчивость при каких-либо $k_{1} \neq k ?$

3. Какие типы $\alpha$ неприводимых представлений групп $S_{n}$ входят в множество $F_{n}(k)$ (см. (2.7)), либо (хотя бы) сколько строк или столбцов содержат соответствующие этим $\alpha$ схемы Юнга, если система $Z_{n}$ является $k$-устойчивой?

4. Остается ли теорема 2.1 справедливой, если всюду в работе вместо множества $E_{n}(k)$ взять $E_{n}\left(k_{2}, k_{1}\right)=E_{n}\left(k_{2}\right) \backslash E_{n}\left(k_{1}\right)\left(k_{2}>k_{1}\right)$ и пространство $B_{n}(k)$ и операторы $H_{n}(k), H_{n}(D ; k)$ заменить соответственно на пространство $B_{n}\left(k_{2}, k_{1}\right)$ и операторы $H_{n}\left(k_{2}, k_{1}\right), H_{n}\left(D ; k_{2}, k_{1}\right)$, определенные с использованием $E_{n}\left(k_{2} ; k_{1}\right)$ вместо $E_{n}(K)$, а понятие $k$-устойчивости заменить на $\left(k_{2}, k_{1}\right)$-устойчивость?

Общая причина того, что сегодня мы не в состоянии дать ответы на эти вопросы заключается в нерешенности задачи о взаимном расположении величин inf $H_{n}^{(\alpha)}$ в зависимости от $\alpha$. Эта труднейшая задача не решена даже для НР-систем: в этом случае результаты имеются лишь для одномерных частиц [8].

\section{3. ДОКАЗАТЕЛЬСТВА}

3.1. Доказательство теоремы 2.1. Пусть $\alpha \in F_{n}(k)$ (см. $\left.(2.7)\right)$ и $\Phi_{n}(\alpha ; Q)=$ $\inf H_{n}^{(\alpha)}(Q)$. Очевидно, $\inf H_{n}^{(\alpha)} \equiv \inf _{Q} \Phi_{n}(\alpha ; Q)$. Так как функция $\Phi_{n}(\alpha ; Q)$ непрерывна по $Q$ и $\inf H_{n}^{(\alpha)}<\mu_{n}(k)$, найдется такое открытое множество $\Gamma_{n}(\alpha) \subseteq \mathbb{R}^{3}$ значений $Q$, что для любого $Q \in \Gamma_{n}(\alpha)$

$$
\inf H_{n}^{(\alpha)}(Q)<\mu_{n}(k) \text {. }
$$

Далее, в силу (1.12a) и $(2.1) \mu_{n}(k) \leqslant \inf H_{n}^{(\alpha)}(D)$ для любых $\alpha \in E_{n}(k)$ и $D$. Как легко видеть,

$$
\inf H_{n}^{(\alpha)}(D)=\inf _{Q^{\prime}} \inf H_{n}^{(\alpha)}\left(Q^{\prime} ; D\right),
$$

и поэтому в силу $(1.126) \mu_{n}(k) \leqslant \inf H_{n}^{(\alpha)}\left(Q^{\prime} ; D\right)$ при каждом $\alpha \in E_{n}(k)$ и любых $D$ и $Q^{\prime}$. Следовательно,

$$
\mu_{n}^{(\alpha)}\left(Q^{\prime}\right):=\min _{D} \inf H_{n}^{(\alpha)}\left(Q^{\prime} ; D\right) \geqslant \mu_{n}(k) .
$$

Согласно [9] $\mu_{n}^{(\alpha)}\left(Q^{\prime}\right)$ есть нижняя граница существенного спектра оператора $H_{n}^{(\alpha)}\left(Q^{\prime}\right)$. Поэтому из неравенств (3.1), (3.2) следует, что при любых $\alpha \in F_{n}(k)$ и $Q \in \Gamma_{n}(\alpha)$ число $\inf H_{n}^{(\alpha)}(Q)$ есть точка дискретного спектра оператора $H_{n}^{(\alpha)}(Q)$. Теорема 2.1 доказана.

Докажем следствие. Из (1.12б) вытекает, что нижняя граница $\mu_{n}\left(Q^{\prime} ; k\right)$ существенного спектра оператора $H_{n}\left(Q^{\prime} ; k\right)$ есть

$$
\mu_{n}\left(Q^{\prime} ; k\right):=\min _{\alpha \in E_{n}(k)} \mu_{n}^{(\alpha)}\left(Q^{\prime}\right) .
$$


Вследствие $(3.2) \mu_{n}\left(Q^{\prime} ; k\right) \geqslant \mu_{n}(k)$, и поэтому все точки спектра оператора $H_{n}\left(Q^{\prime} ; k\right)$, лежащие слева от $\mu_{n}(k)$, принадлежат его дискретному спектру. Пусть $\Gamma_{n}[k]=$ $\bigcup_{\alpha \in F_{n}(k)} \Gamma_{n}(\alpha)$. Если $Q \in \Gamma_{n}[k]$, то $Q \in \Gamma_{n}(\alpha)$ для какого-либо $\alpha \in F_{n}(k)$, и, значит,

$$
\lambda:=\inf H_{n}^{(\alpha)}(Q)<\mu_{n}(k) .
$$

Поэтому $\lambda \in s_{\mathrm{d}}\left(H_{n}(Q ; k)\right)$, и следствие доказано.

3.2. Доказательство теоремы 2.2. Предположим, что теорема неверна, т.е. что для некоторого $n_{0}>0$ система $Z_{n} k$-неустойчива при любых $n \geqslant n_{0}$. Фиксируем $n \geqslant n_{0}$. Тогда найдется такое разбиение $D^{\prime}=\left\{C_{1}^{\prime}, \ldots, C_{m}^{\prime}\right\}$ системы $Z_{n}$, что выполняется неравенство

$$
\inf H_{n}(k) \geqslant \inf H_{n}\left(D^{\prime} ; k\right) \text {. }
$$

Для произвольного кластера $C \subset Z_{n}$ обозначим через $|C|$ число элементов $C$, через $S[C]$ - группу перестановок частиц из $C$. Ясно, что группа $S[C]$ изоморфна группе $S_{|C|}$. Пусть

$$
\breve{S}\left(D^{\prime}\right)=S\left[C_{1}^{\prime}\right] \times S\left[C_{2}^{\prime}\right] \times \cdots \times S\left[C_{m}^{\prime}\right]
$$

и $I_{n}\left(\alpha ; D^{\prime}\right)$ - множество всех типов $\breve{\alpha}=\breve{\alpha}\left(D^{\prime}\right)$ неприводимых представлений группы $\breve{S}\left(D^{\prime}\right)$, содержащихся в представлении типа $\alpha$ группы $S_{n}$ после ограничения его с $S_{n}$ на $\breve{S}\left(D^{\prime}\right)$. Для любого $\breve{\alpha} \in I_{n}\left(\alpha ; D^{\prime}\right)$ обозначим через $P_{n}^{(\breve{\alpha})}\left(D^{\prime}\right)$ проектор в $\mathcal{L}_{2}\left(\mathbb{R}^{3 n}\right)$ на подпространство функций, преобразующихся под действием операторов $T_{g}, T_{g} \psi(r)=\psi\left(g^{-1} r\right), g \in \breve{S}\left(D^{\prime}\right)$, по представлению типа $\breve{\alpha}$, и положим $B_{n}^{(\breve{\alpha})}=P_{n}^{(\breve{\alpha})}\left(D^{\prime}\right) \mathcal{L}_{2}\left(\mathbb{R}^{3 n}\right)$. Очевидно,

$$
P_{n}^{(\alpha)} \mathcal{L}_{2}\left(\mathbb{R}^{3 n}\right)=\sum_{\breve{\alpha} \in I_{n}\left(\alpha ; D^{\prime}\right)} \bigoplus B_{n}^{(\breve{\alpha})},
$$

и, значит,

$$
B_{n}(k)=\sum_{\alpha \in E_{n}(k)} \sum_{\breve{\alpha} \in I_{n}\left(\alpha ; D^{\prime}\right)} \bigoplus B_{n}^{(\breve{\alpha})} .
$$

Пространство $B_{n}^{(\breve{\alpha})}$ инвариантно для оператора $H_{n}\left(D^{\prime}\right)$. Поэтому

$$
\inf H_{n}\left(D^{\prime} ; k\right)=\min _{\alpha \in E_{n}(k) \breve{\alpha} \in I_{n}\left(\alpha ; D^{\prime}\right)} \inf P_{n}^{(\breve{\alpha})} H_{n}\left(D^{\prime}\right) .
$$

Следовательно, найдутся такие $\alpha_{0} \in E_{n}(k)$ и $\breve{\alpha}_{0} \in I_{n}\left(\alpha_{0} ; D^{\prime}\right)$, что

$$
\inf H_{n}\left(D^{\prime} ; k\right)=\inf P_{n}^{\left(\breve{\alpha}_{0}\right)} H_{n}\left(D^{\prime}\right) .
$$

3.3. В силу (3.4) любое неприводимое представление группы $\breve{S}\left(D^{\prime}\right)$ есть тензорное произведение некоторых неприводимых представлений групп $S\left[C_{i}^{\prime}\right], \quad i=$ $1,2, \ldots, m$. Отсюда следует, что для типа $\alpha_{0}$ мы можем указать такие типы $\beta_{i}$ неприводимых представлений групп $S\left[C_{i}^{\prime}\right]$, что

$$
P_{n}^{(\breve{\alpha})}=\prod_{i=1}^{m} P^{\left(\beta_{i}\right)}\left[C_{i}^{\prime}\right],
$$


где $P^{\left(\beta_{i}\right)}\left[C_{i}^{\prime}\right]$ - проекторы, определенные аналогично проекторам $P_{n}^{(\alpha)}$, но действующие только на переменные $r_{j}, j \in C_{i}^{\prime}, i=1, \ldots, m$. Поэтому в силу (1.7) (с $D=D^{\prime}$ )

$$
P_{n}^{(\breve{\alpha})} H_{n}\left(D^{\prime}\right)=\sum_{i=1}^{m} H^{\left(\beta_{i}\right)}\left[C_{i}^{\prime}\right],
$$

где $H^{\left(\beta_{i}\right)}\left[C_{i}^{\prime}\right]=P^{\left(\beta_{i}\right)}\left[C_{i}\right] H\left[C_{i}\right]$.

По построению $\beta_{i}$ есть один из типов неприводимых представлений группы $S\left[C_{i}^{\prime}\right]$, которые возможны для нее после ограничения неприводимого представления типа $\alpha_{0}$ группы $S_{n}$ на $S\left[C_{i}^{\prime}\right]$. Отсюда вытекает [4], [6], что типу $\beta_{i}$ отвечает схема Юнга $Y\left(\beta_{i}\right)$ из $t_{i}=\left|C_{i}^{\prime}\right|$ клеток, полученная из отвечающей типу $\alpha_{0}$ схемы Юнга $Y\left(\alpha_{0}\right)$ удалением $n-t_{i}$ "крайних" клеток из соответствующих строк или столбцов. Следовательно, схема $Y\left(\beta_{i}\right)$ не может содержать больше столбцов и строк, чем $Y\left(\alpha_{0}\right)$, т.е. $\beta_{i} \in E_{t_{i}}(k)$. Из этого факта и из того, что операторы $H\left[C_{i}^{\prime}\right]$ и $H_{t_{i}}$ унитарно эквивалентны, имеем $\left.{ }^{4}\right)$

$$
\inf H^{\left(\beta_{i}\right)}\left[C_{i}^{\prime}\right] \geqslant \inf H_{t_{i}}(k)
$$

В силу (3.3), (3.5)-(3.7)

$$
\inf H_{n}(k) \geqslant \sum_{i=1}^{m} \inf H_{t_{i}}(k)
$$

где, очевидно, $\sum_{i=1}^{m} t_{i}=n$.

3.4. Если для некоторого $i$ выполняется неравенство $t_{i} \geqslant n_{0}$, то по предположению п. 3.2 система $t_{i}$ частиц $k$-неустойчива. Применяя к этой системе подход, который в п. 3.3 был применен к системе $Z_{n}$, убеждаемся в существовании такого разбиения $t_{i}=t_{i 1}+t_{i 2}+\cdots+t_{i s_{i}}$ числа $t_{i}$, что справедлива аналогичная (3.8) оценка

$$
\inf H_{t_{i}}(k) \geqslant \sum_{j=1}^{s_{i}} \inf H_{t_{i j}}(k),
$$

где $1 \leqslant t_{i j}<t_{i}, s_{i} \geqslant 2$.

Применяя этот подход сначала для всех операторов $H_{t_{i}}(k), t_{i} \geqslant n_{0}$, потом для всех операторов $H_{t_{i j}}(k), t_{i j} \geqslant n_{0}$, и т.д. и подставляя полученные оценки в (3.8), мы получим после конечного числа шагов неравенство

$$
\inf H_{n}(k) \geqslant \sum_{i=1}^{p} \inf H_{n_{i}}(k),
$$

где $n_{i}<n_{0}, i=1,2, \ldots, p, \sum_{i=1}^{p} n_{i}=n$.

Пусть $d_{1}=\min _{l<n_{0}} \inf H_{l}(k)$. Тогда в силу (3.10) для любого $n \geqslant n_{0}$ выполняется соотношение

$$
\inf H_{n}(k) \geqslant-n\left|d_{1}\right|
$$

\footnotetext{
4) Нас не должен беспокоить случай $t_{i}<k$, так как величина $k$ ограничивает число столбцов (или строк) в возможных схемах Юнга лишь сверху.
} 
3.5. Далее мы для каждого $n \gg 1$ построим такую нормированную функцию $\widetilde{\psi}_{n}(r)$ из пространства $B_{n}(k)$, что

$$
\left(H_{n}(k) \widetilde{\psi}_{n}, \widetilde{\psi}_{n}\right) \leqslant-d_{2} n^{2}
$$

где $d_{2}>0, d_{2}$ не зависит от $n$. Тогда в силу (3.11), (3.12) при всех $n, n \geqslant n_{0}, n \gg 1$, будет выполнена оценка

$$
-\left|d_{1}\right| n^{-1} \leqslant-d_{2}
$$

которая справедлива для всех $n$ лишь при $d_{2} \leqslant 0$. Но $d_{2}>0$. Из полученного противоречия будет вытекать, что неравенство (3.11) не имеет места, поэтому лежащее в основе его вывода предположение о $k$-неустойчивости систем $Z_{n}$ при всех $n \geqslant n_{0}$ для какого-либо $n_{0}$ неверно. Отсюда будет следовать первое утверждение теоремы 2.2. Второе утверждение, неравенство (2.10), будет доказано в п. 3.11.

3.6. Переходим к построению функций $\widetilde{\psi}_{n}(r)$, для которых верно неравенство (3.12). Пусть $\varphi_{t}(u), u \in \mathbb{R}^{3}, t=1,2, \ldots, n,-$ финитные ортонормированные в $\mathbb{R}^{3}$ функции из $W_{2}^{1}\left(\mathbb{R}^{3}\right)$. Положим

$$
\psi_{n}(r)=\prod_{t=1}^{n} \varphi_{t}\left(r_{t}\right)
$$

Пусть $\chi_{g}^{(\alpha)}$ и $l_{\alpha}-$ характер и размерность представления типа $\alpha \in E_{n}(k)$. Как известно [6], проекторы $P_{n}^{(\alpha)}$ (см. п. 1.5) имеют вид

$$
P_{n}^{(\alpha)} \psi(r)=\frac{l_{\alpha}}{n !} \sum_{g \in S_{n}} \bar{\chi}_{g}^{(\alpha)} \psi\left(g^{-1} r\right) .
$$

Положим $\widetilde{\psi}_{n}(r)=\left\|P_{n}^{(\alpha)} \psi_{n}\right\|^{-1} P_{n}^{(\alpha)} \psi_{n}$ и докажем, что при подходящем выборе функций $\varphi_{t}\left(r_{t}\right)$ неравенство (3.12) будет выполняться. Нам необходимо оценить сверху величины $\left(P_{n}^{(\alpha)} \psi_{n}, P_{n}^{(\alpha)} \psi_{n}\right),\left(K_{n} P_{n}^{(\alpha)} \psi_{n}, P_{n}^{(\alpha)} \psi_{n}\right)$ и $\left(V_{n} P_{n}^{(\alpha)} \psi_{n}, P_{n}^{(\alpha)} \psi_{n}\right)$, для чего полезны следующие соотношения:

$$
\begin{aligned}
& \left(\psi_{n}(r), \psi_{n}\left(g^{-1} r\right)\right)=0, \quad g \neq e, \quad g \in S_{n}, \\
& \left(K\left(r_{i}\right) \psi_{n}(r), \psi_{n}\left(g^{-1} r\right)\right)=0, \quad g \neq e, \quad g \in S_{n}, \quad i=1, \ldots, n, \\
& \left(V\left(\left|r_{i}-r_{j}\right|\right) \psi_{n}(r), \psi_{n}\left(g^{-1} r\right)\right)=0, \quad g \neq e, \quad g \neq g_{i j}, \quad g \in S_{n},
\end{aligned}
$$

где $e$ - единичный элемент $S_{n}, g_{i j}=\left(\begin{array}{cc}i & j \\ j & i\end{array}\right) \in S_{n}$.

Докажем эти соотношения. Пусть

$$
g^{-1}=\left(\begin{array}{cccc}
1 & 2 & \ldots & n \\
t_{1} & t_{2} & \ldots & t_{n}
\end{array}\right) .
$$

Тогда, очевидно,

$$
\psi_{n}\left(g^{-1} r\right)=\prod_{s=1}^{n} \varphi_{t_{s}}\left(r_{s}\right)
$$


Если $g \neq e$, то найдутся $p, q$ такие, что $t_{p} \neq p, t_{q} \neq q$, и (3.13) будет выполняться, например, как следствие равенства

$$
\left(\varphi_{p}\left(r_{p}\right), \varphi_{t_{p}}\left(r_{p}\right)\right)_{\mathcal{L}_{2}\left(\mathbb{R}^{3}\right)}=0 .
$$

Далее, поскольку оператор $K\left(r_{i}\right)$ действует только на функцию $\varphi_{i}\left(r_{i}\right)$, то $(3.14)$ при $p \neq i$ будет следовать из (3.17), а при $p=i-$ из равенства

$$
\left(\varphi_{q}\left(r_{q}\right), \varphi_{t_{q}}\left(r_{q}\right)\right)_{\mathcal{L}_{2}\left(\mathbb{R}^{3}\right)}=0
$$

Теперь проверим (3.15). Так как $g \neq g_{i j}$, то $g^{-1} \neq g_{i j}$. Пусть сначала в (3.16) $t_{i}=i, t_{j}=j$ или $t_{i}=j, t_{j}=i$. Так как $g \neq e, g \neq g_{i j}$, в рассматриваемом случае существует $p \neq i, j$ такое, что $t_{p} \neq p, i, j$. Поэтому будет верно (3.17), а следовательно, и (3.15). Если $t_{i}=i, t_{j}=q \neq i, j$, или $t_{i}=j, t_{j}=q \neq i, j$, или $t_{i}=q \neq i, j$, то $t_{q} \neq q$. Поэтому будет верно (3.18), а значит, и (3.15). Таким образом равенства (3.13)-(3.15) доказаны.

3.7. Из (3.13)-(3.15), поскольку $P_{n}^{(\alpha)} K_{n}(r)=K_{n}(r) P_{n}^{(\alpha)}, P_{n}^{(\alpha)} V_{n}(r)=V_{n}(r) P_{n}^{(\alpha)}$, имеем

$$
\begin{aligned}
\left(P_{n}^{(\alpha)} \psi_{n}, P_{n}^{(\alpha)} \psi_{n}\right) & =\left(\psi_{n}, P_{n}^{(\alpha)} \psi_{n}\right)=\frac{l_{\alpha}^{2}}{n !}\left\|\psi_{n}\right\|^{2}=\frac{l_{\alpha}^{2}}{n !}, \\
\left(K_{n} P_{n}^{(\alpha)} \psi_{n}, P_{n}^{(\alpha)} \psi_{n}\right) & =\left(K_{n} \psi_{n}, P_{n}^{(\alpha)} \psi_{n}\right)=\frac{l_{\alpha}^{2}}{n !}\left(K_{n} \psi_{n}, \psi_{n}\right), \\
\left(V_{n} P_{n}^{(\alpha)} \psi_{n}, P_{n}^{(\alpha)} \psi_{n}\right) & =\left(V_{n} \psi_{n}, P_{n}^{(\alpha)} \psi_{n}\right)= \\
& =\sum_{\substack{s, t=1 \\
s<t}}^{n}\left(V\left(\left|r_{s}-r_{t}\right|\right) \psi_{n}, l_{\alpha} \psi_{n}+\bar{\chi}_{g_{s t}}^{(\alpha)} \psi\left(g_{s t} r\right)\right) \frac{l_{\alpha}}{n !}
\end{aligned}
$$

Отсюда, полагая $\widetilde{\psi}_{n}=\left\|P_{n}^{(\alpha)} \psi_{n}\right\|^{-1} P_{n}^{(\alpha)} \psi_{n}$, получим равенства

$$
\begin{aligned}
\left(K_{n} \widetilde{\psi}_{n}, \widetilde{\psi}_{n}\right) & =\left(K_{n} \psi_{n}, \psi_{n}\right), \\
\left(V_{n} \widetilde{\psi}_{n}, \widetilde{\psi}_{n}\right) & =\sum_{\substack{s, t=1, s<t}}^{p} J_{s t},
\end{aligned}
$$

где

$$
J_{s t}=\left(V\left(\left|r_{s}-r_{t}\right|\right) \psi_{n}, \psi_{n}+\theta_{n}(\alpha) \psi_{n}\left(g_{s t} r\right)\right), \quad \theta_{n}(\alpha)=\frac{1}{l_{\alpha}} \bar{\chi}_{g_{s t}}^{(\alpha)} .
$$

3.8. Далее определим функции $\varphi_{t}\left(r_{t}\right)$ (см. п. 3.6) так же, как в [1]. А именно, пусть число $A$ таково, что выполняется неравенство $(2.8), p \geqslant 1$ и $\omega \gg 1$ - целые числа. Положим

$$
\begin{gathered}
f_{p}\left(x_{1}\right)=\frac{1}{\sqrt{A}} \sin \frac{2 \pi p \omega}{A} x_{1} \quad \text { при } \quad\left|x_{1}\right| \leqslant A, \quad f_{p}\left(x_{1}\right) \equiv 0 \quad \text { при }\left|x_{1}\right| \geqslant A, \\
f_{i j k}\left(r_{s}\right)=f_{i}\left(x_{s}\right) f_{j}\left(y_{s}\right) f_{k}\left(z_{s}\right), \quad s=1,2, \ldots, n .
\end{gathered}
$$


Для каждого $n$ укажем число $\bar{n}$ такое, что $\bar{n}^{3} \leqslant n<(\bar{n}+1)^{3}$, и выберем тройки натуральных чисел $i_{t}, j_{t}, k_{t}$ так, что $1 \leqslant i_{t}, j_{t}, k_{t} \leqslant \bar{n}+1,\left(i_{t}, j_{t}, k_{t}\right) \neq\left(i_{s}, j_{s}, k_{s}\right)$ при $t \neq s$. Пусть

$$
\varphi_{t}\left(r_{t}\right)=f_{i_{t} j_{t} k_{t}}\left(r_{t}\right), \quad \psi_{n}=\prod_{t=1}^{n} \varphi_{t}\left(r_{t}\right) .
$$

Тогда в силу неравенств (2.9) из работы [1] и формулы (3.19)

$$
\left(K_{n} \widetilde{\psi}_{n}, \widetilde{\psi}_{n}\right) \leqslant c n^{5 / 3}
$$

где $c$ - константа, не зависящая от $n$.

3.9. Оценим теперь произвольное слагаемое $J_{s t}$ в сумме (3.20). Очевидно,

$$
J_{s t}=J_{s t}^{0}+\theta_{n}(\alpha) J_{s t}^{1}
$$

где

$$
\begin{aligned}
J_{s t}^{0} & =\int_{r_{s}, r_{t} \in C(A)} V\left(\left|r_{s}-r_{t}\right|\right) \varphi_{s}^{2}\left(r_{s}\right) \varphi_{t}^{2}\left(r_{t}\right) d r_{s} d r_{t} \\
J_{s t}^{1} & =\int_{r_{s}, r_{t} \in C(A)} V\left(\left|r_{s}-r_{t}\right|\right) \varphi_{s}\left(r_{s}\right) \varphi_{t}\left(r_{t}\right) \varphi_{s}\left(r_{t}\right) \varphi_{t}\left(r_{s}\right) d r_{s} d r_{t}
\end{aligned}
$$

Оценим сначала величину $\theta(\alpha)$. Пусть $\alpha=\alpha\left(n_{1}, \ldots, n_{p}\right)$ (см. п. 1.5). Тогда согласно равенству (5.6) из [4]

$$
\theta_{n}(\alpha) \equiv \frac{1}{l_{\alpha}} \chi_{g_{s t}}^{(\alpha)}=\sum_{m=1}^{p} \frac{n_{m}\left(n_{m}-2 m+1\right)}{n(n-1)},
$$

где $\sum_{m=1}^{p} n_{m} \equiv n, n_{m} \geqslant 1$. Так как $\alpha \in E_{n}(k)$, то либо $p \leqslant k$ (случай бозонов), либо $n_{m} \leqslant k, m=1 \ldots p$ (случай фермионов). Поэтому при фиксированном $k$

$$
\sup _{n} \sup _{\alpha \in E_{n}(k)}\left|\theta_{n}(\alpha)\right|<+\infty
$$

Далее заметим, что функционалы $J_{s p}^{j}, j=0,1$, зависят от параметра $\omega$, входящего в функции $\varphi_{t}$ и $\varphi_{s}$ (см. п. 3.8). Согласно [1] при большом $\omega$ и всех $n$

$$
J_{s t}^{0}=J_{s t}^{0}(\omega) \leqslant a \delta
$$

где число $a>0$ и не зависит ни от $n$, ни от $\omega$.

3.10. Для оценки $J_{s t}^{1}=J_{s t}^{1}(\omega)$ воспользуемся тем, что при $r_{s}, r_{t} \in C(A)$

$$
\begin{aligned}
W_{s t}\left(r_{s}, r_{t}, \omega\right) & :=\varphi_{s}\left(r_{s}\right) \varphi_{t}\left(r_{s}\right) \varphi_{s}\left(r_{t}\right) \varphi_{t}\left(r_{t}\right)= \\
& =\frac{1}{(2 A)^{6}} \prod_{m=1}^{6}\left(\cos \frac{2 \pi d_{m}^{-} \omega \xi_{m}}{A}-\cos \frac{2 \pi d_{m}^{+} \omega \xi_{m}}{A}\right)
\end{aligned}
$$


где $d_{1}^{ \pm}=\left(i_{s} \pm i_{t}\right), d_{2}^{ \pm}=\left(j_{s} \pm j_{t}\right), d_{3}^{ \pm}=\left(k_{s} \pm k_{t}\right), d_{m}^{ \pm}=d_{m-3}^{ \pm}, m=4,5,6, \xi_{1}=x_{s}$, $\xi_{2}=y_{s}, \xi_{3}=z_{s}, \xi_{4}=x_{t}, \xi_{5}=y_{t}, \xi_{6}=z_{t}$. Поэтому

$$
W_{s t}\left(r_{s}, r_{t}, \omega\right)=\sum_{l=1}^{64} W_{l}\left(r_{s}, r_{t}, \omega\right)
$$

где

$$
W_{l}\left(r_{s}, r_{t}, \omega\right)=\frac{\gamma_{l}}{(2 A)^{6}} \prod_{m=1}^{6} \cos \frac{2 \pi}{A} d_{l m} \omega \xi_{m}, \quad \gamma_{l}= \pm 1, \quad d_{l m}=d_{m}^{ \pm}
$$

По построению $\left(i_{s}, j_{s}, k_{s}\right) \neq\left(i_{t}, j_{t}, k_{t}\right)$, следовательно, для каждого $l$ найдется такое $m_{0}=m_{0}(l)$, что $\left|d_{l m_{0}}\right| \geqslant 1$. Учитывая это и то, что $V\left(\left|r_{1}\right|\right) \in \mathcal{L}_{2, \text { loc }}\left(\mathbb{R}^{3}\right)$, имеем

$$
\int_{r_{s}, r_{t} \in C(A)} V\left(\left|r_{s}-r_{t}\right|\right) W_{l}\left(r_{s}, r_{t}, \omega\right) d r_{s} d r_{t} \rightarrow 0 \quad \text { при } \quad \omega \rightarrow \infty
$$

в силу неравенства Бесселя. Отсюда и из (3.23) вытекает, что при больших $\omega$

$$
\left|\theta_{n}(\alpha) J_{s t}^{1}(\omega)\right| \leqslant \frac{a}{2}|\delta|
$$

и в силу (3.24) справедливо неравенство $J_{s t}(\omega)<a \delta / 2$. Таким образом, из (3.20) следует, что

$$
\left(V_{n} \widetilde{\psi}_{n}, \widetilde{\psi}_{n}\right) \leqslant c_{0} n^{2} \delta
$$

где $c_{0}>0$ и не зависит от $n$.

Из неравенств (3.21) и (3.26) вытекает (3.12), и тем самым первое утверждение теоремы 2.2 доказано.

3.11. Итак, мы доказали существование такой последовательности чисел $n_{1}<$ $n_{2}<\cdots<n_{s}<n_{s+1}<\cdots$, что система $Z_{n_{s}} k$-устойчива, $s=1,2, \ldots$. Не ограничивая общности, считаем, что $n_{s}<n_{s+1}-1$. Тогда система из $N=n_{s+1}-1$ частищ будет $k$-неустойчива. Повторяя рассуждения п. $3.3,3.4$ с подстановкой $n_{s}$ вместо $n_{0}$ и учитывая (3.12), (3.9), мы придем к оценке

$$
-d_{2} N^{2} \geqslant \inf H_{N}(k) \geqslant \sum_{i=1}^{m} \inf H_{t_{i}}(k),
$$

где $\sum_{i=1}^{m} t_{i}=N, t_{i} \leqslant n_{s}$. Вследствие $(2.9)$

$$
\inf H_{t_{i}}(k) \geqslant-d_{0} t_{i}^{2}
$$

В силу (3.27), (3.28)

$$
N^{2} \leqslant d_{3} \sum_{i=1}^{m} t_{i}^{2}
$$

где $d_{3}>0$ и не зависит от $m, N$ и $n_{s}$. Здесь $\sum_{i=1}^{m} t_{i}=N, t_{i} \leqslant n_{s}, i=1,2, \ldots, m$. 
Пусть теперь $t_{p}=\min _{i} t_{i}$ и $t_{j}<n_{s}, j \neq p$. Построим новое разбиение $\tau^{\prime}=$ $\left(t_{1}^{\prime}, \ldots, t_{m}^{\prime}\right)$, полагая $t_{i}^{\prime}=t_{i}, i \neq p, j, t_{p}^{\prime}=t_{p}-1, t_{j}^{\prime}=t_{j}+1$. Очевидно, что в разбиении $\tau^{\prime}$ и в некоторых других в последующих рассуждениях возможно обращение в нуль отдельных слагаемых (в $\tau^{\prime}$ имеем $t_{p}^{\prime}=0$ при $\left.t_{p}=1\right)$. Ясно, что для разбиения $\tau^{\prime}$

$$
\sum_{i=1}^{m}\left(t_{i}^{\prime}\right)^{2} \geqslant \sum_{i=1}^{m} t_{i}^{2}, \quad \sum_{i=1}^{m} t_{i}^{\prime}=N, \quad t_{i}^{\prime} \leqslant n_{s}, \quad i=1,2, \ldots, m .
$$

Пусть далее $t_{p^{\prime}}^{\prime}=\min _{i, t_{i}^{\prime}>0} t_{i}^{\prime}$ и $t_{j^{\prime}}^{\prime} \neq 0, t_{j}^{\prime}<n_{s}, j^{\prime} \neq p^{\prime}$. Построим разбиение $\tau^{\prime \prime}=\left(t_{1}^{\prime \prime}, \ldots, t_{m}^{\prime \prime}\right)$, где $t_{i}^{\prime \prime}=t_{i}^{\prime}$ при $i \neq p^{\prime}, j^{\prime}, t_{p^{\prime}}^{\prime \prime}=t_{p^{\prime}}^{\prime}-1, t_{j^{\prime}}^{\prime \prime}=t_{j^{\prime}}^{\prime}+1$.

Тогда, очевидно,

$$
\sum_{i=1}^{m}\left(t_{i}^{\prime \prime}\right)^{2} \geqslant \sum_{i=1}^{m}\left(t_{i}^{\prime}\right)^{2}, \quad \sum_{i=1}^{m} t_{i}^{\prime \prime}=N, \quad t_{i}^{\prime \prime} \leqslant n_{s} .
$$

Продолжая подобные рассуждения, мы после конечного числа шагов придем к разбиению $\tilde{\tau}=\left(\tilde{t}_{1}, \ldots, \tilde{t}_{m_{1}}\right)$, для которого

$$
\sum_{i=1}^{m_{1}} \tilde{t}_{i}^{2} \geqslant \sum_{i=1}^{m} t_{i}^{2}
$$

и все числа $\tilde{t}_{i}$, кроме, быть может, одного, скажем $\tilde{t}_{m_{1}}$, равны $n_{s}, 0 \leqslant \tilde{t}_{m_{1}} \leqslant n_{s}$, a $\left(m_{1}-1\right) n_{s}+\tilde{t}_{m_{1}}=N$. Отсюда следует, что $m_{1} \leqslant N n_{s}^{-1}+1$, поэтому в силу (3.29), (3.30)

$$
N^{2} \leqslant d_{3} n_{s}^{2}\left(N n_{s}^{-1}+1\right) .
$$

Из данного неравенства вытекает (2.10).

Благодарности. Работа поддержана РФФИ (грант № 05-01-00299).

\section{Список литературы}

[1] Г. М. Жислин, ТМФ, 152:3 (2007), 528-537.

[2] С. А. Вугальтер, Г. М. Жислин, ТМФ, 76:1 (1988), 132-142.

[3] R. Lewis, H. Siedentop, S. A. Vugalter, Ann. Inst. H. Poincaré Phys. Théor., 67:1 (1997), $1-28$.

[4] Ф. Мурнаган, Теория представлений групп, ИЛ, М., 1950.

[5] М. Хамермеш, Теория групп и ее применение к физическим проблемам, Мир, М., 1966.

[6] Е. Вигнер, Теория групп и ее приложение $к$ квантомеханической теории атомных спектров, ИЛ, М., 1961.

[7] С. А. Вугальтер, Г. М. Жислин, ТМФ, 121:2 (1999), 297-306.

[8] E. Lieb, D. Matiss, J. Math. Phys., 3 (1962), 749-751.

[9] G. M. Zhislin, SIGMA, 2 (2006), 024; arXiv: math-ph/0602050.

Поступила в редакцию 28.12.2007

5 Теоретическая и математическая физика, т. 157, № 1, 2008 г. 